\title{
TIME EVOLUTION OF BEAM CURRENT IN THE RECYCLER RING
}

\author{
K. Gounder*, J. Marriner and S. Mishra \\ FNAL, Batavia, IL 60510, USA.
}

\begin{abstract}
We study the time evolution of the beam current in the Fermilab Recycler Ring due to abrupt physical processes (single coloumb scattering, nuclear scattering) that cause sudden loss of beam, and diffusive processes (multiple coloumb scattering, lattice dpendence, etc.) which cause emittance growth. This emittance growth combined with finite aperure of the beam pipe will lead to eventual loss of most beam. We develop a fitting technique to the time evolution of beam current to estimate emittance growth. Finally we compare the directly measured growth with the fitted value. Work supported by the U.S. Department of Energy under contract No. DE-AC02-76CH03000.
\end{abstract}

\section{INTRODUCTION}

The Recycler Ring [1] located in the Main Injector tunnel at Fermilab is designed to store antiprotons from the Accumulator and the residual Tevatron stores as a part of Run II luminosity upgrade program. The Recycler Ring (RR) is expected to improve the luminosity by increasing the antiproton accumulation efficiency and recycling the residual Tevatron antiprotons after colliding stores [2]. Presently, the RR is being commissioned using protons as well as antiprotons. The successful operation of the Ring requires a beam lifetime of $>100$ hours with stochastic cooling and high stacking efficiency. Therefore the study of beam evolution and emittance growth rate is essential for the RR to be an efficient storage ring. The basic parameters for RR are listed in Table 1.

\begin{tabular}{|l|c|}
\hline Paramater & Value \\
\hline Acceptance $(\mathrm{mm}-\mathrm{mr})$ & $40.0 \pi$ \\
Average $\beta(\mathrm{m})$ & 42.0 \\
Average beam pipe radius (in $\mathrm{m})$ & 0.023 \\
Beam energy $(\mathrm{GeV})$ & 8.89 \\
Average beam $\beta$ & 0.998 \\
Average beam $\gamma$ & 9.48 \\
Maximum energy loss $(\mathrm{GeV})$ & 0.089 \\
\hline
\end{tabular}

Table 1: The basic Recycler Ring parameters relevant for the computations detailed in this note.

\section{BASIC FORMULATION}

For most lifetime measurements, we introduce a thin approximately Gaussian beam of known intensity in the mid-

\footnotetext{
*gounder@fnal.gov
}

dle of RR aperture. In such cases, the beam lifetime and evolution are determined by two classes of processes: (1) Processes like single coloumb scattering, nuclear scattering or ionization by which the beam loses a particle abruptly at any given time and region of the beam; (2) Diffusion processes like multiple coloumb scattering, intrabeam scattering or some form of noise where the beam emittance grows and eventually the beam loses particles by hitting the aperture of the beam pipe. Assuming these two classes of processes are independent [6], we write the beam current at any given time as:

$$
I(t)=I_{0} N_{a b}(t) N_{d f}(t)
$$

where $I_{0}$ is the initial beam introduced, $N_{a b}(t)$ denotes time evolution due to abrupt loss of beam particles as in the first case, and $N_{d f}(t)$ denotes the time evolution due to diffusion processes as described in the second case. For most cases, we can write:

$$
N_{a b}(t)=e^{-\frac{t}{\tau_{a b}}}
$$

where $\tau_{a b}$ characterizes the lifetime due to processes belong to the first case and assumed to be constant during the evolution. To describe the diffusive processes, we have to solve the Foker-Planck equation [3]:

$$
\frac{\partial f}{\partial \tau}=\frac{\partial}{\partial Z}\left(Z \frac{\partial f}{\partial Z}\right)
$$

where $f$ describes the particle distribution and subject to the boundary conditions:

$$
\begin{gathered}
f(Z, 0)=f_{0}(Z) \\
f(1, \tau)=0 \\
\left.\frac{\partial f}{\partial z}\right|_{z=0}=0
\end{gathered}
$$

where $\mathrm{Z}=\epsilon / \epsilon_{a}=$ emittance/acceptance, and $\tau=\mathrm{tR} / \epsilon_{a}$ with $\mathrm{R}$, the diffusion coefficient. Here $f_{0}$ denotes the beam distribution at $t=0$. In the case of pure multiple coloumb scattering phenomena, the diffusion coefficient $\mathrm{R}$ is given in terms of the mean scattering angle $\theta$ by:

$$
R=\beta_{\text {avg }}\left\langle\dot{\theta}^{2}\right\rangle
$$

The general solution of the above equation can be written as:

$$
f(Z, \tau)=\sum_{n} C_{n} J_{0}\left(\lambda_{n} \sqrt{Z}\right) e^{-\lambda_{n}^{2} \tau / 4}
$$


with coefficients $C_{n}$ :

$$
C_{n}=\frac{1}{J_{1}\left(\lambda_{n}\right)^{2}} \int_{0}^{1} f_{0}(Z) J_{0}\left(\lambda_{n} \sqrt{Z}\right) d Z
$$

where $\lambda_{n}$ is nth root of the Bessel function $J_{0}(Z)$. We obtain the total beam particles as a function of time:

$N_{d f}(t)=\int_{0}^{1} f(Z, \tau) d Z=2 \sum_{n} \frac{C_{n}}{\lambda_{n}} J_{1}\left(\lambda_{n}\right) e^{-\left(\lambda_{n}^{2} R / 4 \epsilon_{a}\right) t}$

The beam lifetime at any time can be computed using:

$$
\tau_{m c}=-\frac{N(\tau)}{d N(\tau) / d \tau}
$$

The beam life time varies with time but reaches an asymptotic value:

$$
\tau_{a}=\frac{4 \epsilon_{a}}{\lambda_{1}^{2} R}
$$

The emittance growth can be obtained from:

$$
\frac{d \epsilon_{N}}{d t}=\frac{\pi \beta \gamma}{2} R
$$

Now combining the expressions for $N_{a b}$ and $N_{d f}$, we cast the time evolution of beam current as:

$$
I(t)=I_{0} e^{-\frac{t}{\tau_{a b}}} 2 \sum_{n} \frac{C_{n}}{\lambda_{n}} J_{1}\left(\lambda_{n}\right) e^{-\left(\lambda_{n}^{2} R / 4 \epsilon_{a}\right) t}
$$

Since the beam current measurement as a function of time is one of the most accurate measurement we can make in the Recycler Ring, we can fit the measurements for two parameters - for the diffusion constant $\mathrm{R}$ and the lifetime due to abrupt processes $\tau_{a b}$. From these, further information about the vacuum residual gas scattering or other processes causing emittance growth can be extracted. This method also provides an alternative to other emittance growth measurements such as using Schottky detectors or techniques based on beam scrapers. Or simply, this could be a cross check on understanding of the relevant physical quantities as well as systematics of other measurement methods.

\section{FITTING PROCEDURE}

To apply the above formalism to real Recycler Ring data, we develop a fitting procedure. From the above formalism, the beam current at a given time $t$ after $t=0$ can be written as:

$$
I(t)=I_{0} e^{-\frac{t}{\tau_{a b}}} \sum_{n} Y_{n} e^{-R \alpha_{n} t}
$$

with the coefficients $Y_{n}=Y_{n}\left(\frac{\sigma}{a}, \epsilon_{a}\right)$, and $\alpha_{n}=\alpha_{n}\left(\epsilon_{a}\right)$. The coefficients $Y_{n}, \alpha_{n}$ can be generated numerically for most cases once knowing the initial beam distribution $\sigma$, the RR acceptance $\epsilon_{a}$ and half aperture a. We can obtain

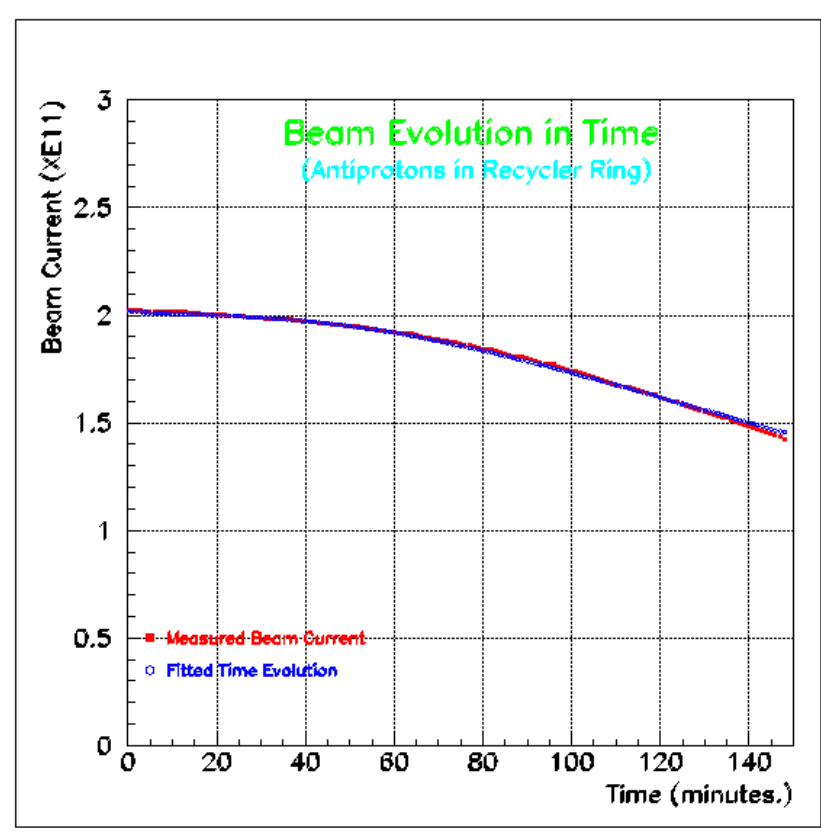

Figure 1: Time evolution of antiproton beam in the Recycler Ring. The dots denote the measured beam current values while the smooth curve is a fit using the first five terms of the expansion for $\mathrm{I}(\mathrm{t})$ as discussed above. The fitted value for the diffusion constant determines the emittance growth rate as $9.75 \pm 2.2 \pi-\mathrm{mm}-\mathrm{mr} /$ hour while the measured value is $9-11 \pi \mathrm{mm}-\mathrm{mr} / \mathrm{hour}$.

$\mathrm{I}(\mathrm{t})$ - from beam current monitors. We can fit $\mathrm{I}(\mathrm{t})$ for $\tau_{a b}$ and $\mathrm{R}$ using any normal fitting technique. Once knowing $\mathrm{R}$, we can extrapolate to many quantities such as computing the emittance growth using:

$$
\frac{d \epsilon_{N}}{d t}=\frac{\pi \beta \gamma}{2} R
$$

For most practical puposes, the sum in the above expression for $\mathrm{I}(\mathrm{t})$ can be limited to first 5 terms or less. This can be easily seen from zeros of the Bessel function $J_{0}(X)$ in increasing order: $2.405,5.520,8.654,11.792$, and 14.931 . Here we use first five terms of the above expansion.

\section{ANTIPROTON BEAM TIME EVOLUTION}

The antiproton beam in the RR is cooled using stochastic cooling methods [4]. The well cooled beam provides an ideal situation for the beam time evolution studies as it has a Gaussian distribution whose initial width can be determined using scraping techniques or a Schottky detec- 
tor. After 2003 January shutdown, the RR vacuum residual gases were unusually dominated by leaks and contamination. An initial Gaussian beam of $2.2 \times 10^{11}$ antiprotons (width $3.7 \mathrm{~mm}$ ) was allowed to evolve in time when no Main Injector ramping was present [5]. The above figure illustrates the beam evolution where the dots denote the beam current measured for 2.5 hours. The data was fitted using the first five terms of the expansion (equation 1) for $\mathrm{I}(\mathrm{t})$ for $\tau_{a b}$ and the diffusion constant $\mathrm{R}$. The fitted values of $\tau_{a b}=37.8 \pm 1.7$ hours $R=0.65 \times 10^{-10}$, $d \epsilon / d t=9.75 \pm 2.2 \pi \mathrm{mm}-\mathrm{mr} /$ hour [7] is consistent with directly measured values of 40 hours and 9-11 $\pi$-mm$\mathrm{mr} /$ hour.

\section{REFERENCES}

[1] Gerry Jackson, “The Fermilab Recycler Ring Technical Design Report”, November 1996, Fermilab-TM-1991; Mishra, "Status of Fermilab Recycler Ring", EPAC2002, Paris, June 2002.

[2] J. Marriner et. al., "Run II Handbook", at http://wwwbd.fnal.gov/runII/index.html. Also see M. Church, "Tevatron Run II Performance and Plans”, EPAC2002, Paris, June 2002.

[3] D. Edwards and M. Syphers, "An Introduction to Physics of High Energy Accelerators", John Wiley and Sons, 1993.

[4] D. Mohl in Advanced Accelerator Physics Course, Proceedings of 1985 CERN Accelerator School, CERN 87-03 (1986).

[5] As the Recycler Ring is located in the MI tunnel, the RR beam is affected by the stray fields while the Main Injector is ramping. More beam pipe shielding and ramping power supplies are installed to minimize this effect.

[6] This is only an aproximation as the threshold scattering angle for single coloumb scattering depends on the size of the beam and beam pipe aperture.

[7] The emittance quoted here is the normalized $95 \%$, i.e., $\epsilon_{N 95 \%}=3.0 \epsilon_{r m s N}$ 